\title{
DELEUZE Y SPINOZA HACIA UNA CONCEPCIÓN ETOLÓGICA DE LA ÉTICA
}

Deleuze and Spinoza towards an ethological conception of ethics

\author{
Eduardo Alberto León \\ Flacso-Ecuador \\ alberto3026@yahoo.es
}

\section{Resumen:}

Este trabajo es una reconstrucción de la ontología materialista de Spinoza utilizando la filosofía de Gilles Deleuze, para enfocarse en una etología. Es por eso que, en primer lugar, se comparará la naturaleza de la ética radical de Spinoza junto con la ética inmanente de Deleuze. Al mismo tiempo, se analizará la filosofía etológica de Spinoza y la noción de agencia (conatus). En la última parte de este artículo, se verá cómo estos conceptos han influido en la formulación de Deleuze y Guattari de una política inmanente del deseo, minoritaria y colectiva. Y por último se explicará cómo esta teoría radical de la ética puede engendrar la diagramación de una política nómada o una revolución por-venir.

\section{Palabas clave:}

Deseo, revolución, etología, ética, inmanencia, etología, conatus

\begin{abstract}
:
This research work is a reconstruction of Spinoza's materialistic ontology using the philosophy of Gilles Deleuze, to focus on an ethology. That is why, first, the nature of Spinoza's radical ethics will be compared along with Deleuze's immanent ethics. At the same time, Spinoza's ethological philosophy and the notion of agency (conatus) will be analyzed. In the last part of this article, we will see how these concepts have influenced the formulation of Deleuze and Guattari of an immanent politics of desire, minoritarian and collective. And finally, it will be explained how this radical theory of ethics can produce the layout of a nomadic policy or a revolution to-come.
\end{abstract}

\section{Keywords:}

Desire, Revolution, Ethology, Ethics, Immanence, Ethology, Conatus 


\section{Introducción}

El Anti-Edipo Capitalismo y Esquizofrenia (2016) de Deleuze y Guattari es uno de los libros más influyentes, provocativos, desconcertantes y contundentes que se han escrito en el campo de la teoría política y la filosofía en el siglo pasado. Algunos autores incluso ven el libro como la contraparte moderna de El anticristo de Friedrich Nietzsche. Es en este libro, donde Deleuze y Guattari sitúan la micropolítica del deseo y el proceso de producción del deseo en el centro de su investigación filosófica, abordan una gama de preguntas desde el psicoanálisis a la política, la economía a la historia, la lingüística a la filosofía con respecto a sociedad capitalista moderna.

No se puede negar que El Anti-Edipo "es un intento de formular una suerte de filosofía política que tuvo el psicoanálisis" (León, 2011: 2). De igual manera, se concibe como un libro revolucionario, es decir, un libro que quiere abrirnos los ojos a la potencia de la revolución en el ámbito de la vida cotidiana. Pero como nos deja en claro que, su definición de revolución no incluye tomar el poder. No significa derrocar un régimen y luego instalar otro régimen y reformar el gobierno de arriba abajo como exigen los maoístas y leninistas. No quiere formar parte de tales programas, en este sentido, Revolución para Deleuze y Guattari significa esquizofrenizar la estructura de poder existente, haciéndola vibrar a un nuevo ritmo, haciéndola cambiar desde dentro, sin que al mismo tiempo se convierta en un esquizofrénico.

En este sentido, el objetivo principal de este artículo, es hacer una reconstrucción de la ontología materialista de Spinoza para luego enfocarse en la ética inmanente del deseo. Es muy importante, en primer lugar, discutir los fundamentos filosóficos de la ontología materialista de Spinoza y el plano de inmanencia propuesto por Deleuze y Guattari, que luego darán lugar a la filosofía etológica de Spinoza y la noción de agencia (conatus). Se verá cómo estos conceptos han influido en la formulación de Deleuze y Guattari de una política inmanente del deseo, minoritaria y colectiva que da lugar a la revolución por-venir ${ }^{1}$, Igualmente es fácil malinterpretar el Anti Edipo si no entendemos a lo que el libro se opone que es la teoría freudiana del deseo, es decir, "el Antiedipo aborrece que el psicoanálisis convierta el deseo en una representación. A juicio de Deleuze, el deseo no puede atribuírsele una representación, porque el inconciente no representa nada, solo produce, la única actividad del inconciente es producir deseo (León, 2011:1). Es por esto, que el concepto desarrollado por Deleuze y Guattari esquizoanálisis enfrenta un serio dilema, ya que su visión se basa en un concepto inherentemente revolucionario del deseo.

\footnotetext{
1 El concepto deleuzeano de «por-venir» no habla ningún futuro -a no ser que esta palabra también sea alterada-. "No se trata de algo que todavía no es pero que se consolida como proyecto para cambiar lo que parece que sí es: el presente, haciéndolo al modo de un «deber ser», esto es, imponiendo una trascendencia en otro tiempo que tendrá que venir. Imponiendo un deber ser abstracto que sirva únicamente para juzgar el presente, sin localizar sus virtualidades, sin dejar lugar a las posibilidades de mundos que ya siempre puede haber si se construyen inmanentemente con los materiales de los que disponemos, sin un "más allá»" (Nunez,2010:108)
} 


\section{Plano de inmanencia y ontología materialista}

Gilles Deleuze describe muy ampliamente en su libro, Spinoza: Filosofía práctica, que para Spinoza "humildad, pobreza y castidad" (Deleuze, 2004: 11) se vuelven de inmediato efectos de una vida particularmente y sobre abundantemente feliz. Spinoza utiliza estas virtudes para no lograr fines morales o caminos religiosos hacia un más allá, puesto que, no hay absolutamente ninguna otra vida para el filósofo. Mientras tanto en, Spinoza y el Problema de la Expresión (1999), Deleuze considera a Spinoza como el príncipe de todos los filósofos. Este hombre merece esta noble descripción porque proporciona "Ios sentimientos, las conductas y las intenciones relacionándolas no a valores trascendentes sino a modos de existencia" (Deleuze, 1999, p. 262). Para ser específicos, Deleuze encuentra en Spinoza la posibilidad radical de pensar la existencia social emancipada de los grilletes del Estado. Esto se puede percibir en el Tratado Teológico Político (2014), donde Spinoza formula una filosofía que evalúa críticamente el statu quo degenerado. Por ejemplo, cuestiona a sus semejantes, por qué eligen su propia esclavitud y la perciben como libertad, un tema convergente problematizado por Deleuze y Guattari en todo El Anti-Edipo: Capitalismo y Esquizofrenia (2016).

La audacia de Spinoza no debería sorprendernos cuando Negri, en Spinoza y Nosotros (2011) lo considera como la anomalía del siglo, una anomalía del materialismo victorioso, de la ontología del ser que siempre avanza y que, al constituirse a sí mismo, plantea la posibilidad ideal de revolucionar el mundo. Aunque la vida puede lograr un cierto grado de optimización dentro de una sociedad democrática o liberal, el filósofo no debe limitarse a sí mismo con eso. Siguiendo a Deleuze, "el filósofo solicita fuerzas en el pensamiento que eluden tanto la obediencia como la culpa, y crea la imagen de más allá del bien y del mal, una inocencia rigurosa sin mérito ni culpabilidad" (Deleuze, 2004: 12). El filósofo puede residir en varios estados, puede frecuentar varios medios, pero lo hace a la manera de un ermitaño, una sombra, un viajero. De esta manera, la disonancia y el nomadismo se legitiman como virtudes cardinales en la filosofía espinozista. En las otras obras de Deleuze, estas virtudes antes mencionadas se conceptualizan de manera análoga. En Nietzsche y Filosofía (2013), concibe las virtudes de la afirmación y la revalorización como los nuevos valores en el trágico mundo de Dionisos que se libera de los grillos del cristianismo.

Una Vida ${ }^{2}$ caracterizada por la fecundidad y el dinamismo es denominada por Spinoza como Naturaleza. Esta importante tesis espinozista depende de la idea de una sola sustancia que tenga modos inestimables de singularidades y atributos, de la misma manera que condiciona la posibilidad de una gran cantidad de devenires. En La Ética, la naturaleza se entiende como sustancia y causa Natura naturans, como efecto y modo Natura naturata ${ }^{3}$. Estas dos condiciones nos permiten hablar de la naturaleza en

${ }^{2}$ La Inmanencia: una vida (2002). Haciendo juego de palabras con "Una Vida"

3 "'Por Natura naturans debemos entender aquello que es en sí y se concibe por sí, o sea, aquellos atributos de la substancia que expresan una esencia eterna e infinita, esto es, Dios [...] Por Natura naturata, en cambio, entiendo aquello que se sigue de la necesidad de la naturaleza de Dios, o sea, de la de cada uno de los atributos de Dios, esto es, todos los modos de los 
general, lo que Spinoza quiere explicar es que la Natura Naturans, como substancia y causa, y la Natura Naturata, "como efecto y modo, se entrelazan en vínculos de mutua inmanencia; por una parte, la causa permanece en sí misma, y por otra, el efecto o el modo permanece en la causa" (Spinozianas: Filosofía de los Márgenes). El naturalismo, en el contexto del espinozismo, satisface la univocidad de los atributos en el que los atributos en la misma forma constituyen la esencia de Dios como naturaleza natural y contienen esencias de modos como la naturaleza ex natura rei la univocidad de la causa. donde la causa de todas las cosas se afirma de Dios como la génesis de la naturaleza natural, es decir, que él mismo se causó; y la univocidad de la modalidad en el cual la necesidad califica tanto el orden de la Natura naturata ${ }^{4}$ como la organización de la Natura naturans.

Con lo dicho anteriormente, el compromiso de Deleuze con Spinoza puede entenderse fructíferamente como la formulación de un problema que surge en el punto de convergencia entre la ética y la ontología, un problema que Deleuze llama etología ${ }^{5}$. A través de este concepto, Deleuze sostiene que la caracterización y evaluación de los modos de vida de un individuo solo se puede hacer desde el punto de vista de una ontología pura.

Spinoza comparte con Duns Scotus la tesis fundamental de la Univocidad del $\mathrm{Ser}^{6}$.La teorización ontológica de Spinoza sobre la univocidad es una valiente antipatía dirigida a una larga historia de tradición filosófica occidental influenciada sistémicamente por la metafísica platónica o la filosofía trascendental. Lo que Deleuze toma de Escoto es la idea de que solo en la univocidad es posible una diferencia

atributos de Dios, en cuanto se los considera como cosas que son en Dios y que sin Dios no pueden ni ser, ni concebirse'. [E1. P.29.esc]

4 “Por naturaleza naturante entendemos un ser que captamos clara y distintamente por sí mismo y sin tener que acudir a algo distinto de él, como todos los atributos que hemos descrito hasta aquí, y ese ser es Dios (...) La naturaleza naturada debemos dividirla en dos: una universal y otra particular. La universal consta de todos los modos que dependen inmediatamente de Dios (...) La naturaleza particular consta de todas las cosas particulares que son causadas por los modos universales. De suerte que la naturaleza naturada necesita algunas substancias [i.e, los atributos de la Etica] para ser correctamente concebida" (Korte Vehandeling van God de Mensch en des Zelfswelstand 1/8).

${ }^{5}$ El proyecto filosófico de Deleuze se caracteriza mejor como la combinación creativa de ontología y ética, es decir, como etología. En este sentido, el primer punto a destacar sobre la concepción ecológica de Deleuze de los seres vivos es que se opone fundamentalmente al individualismo metodológico de cualquier tipo. Un individuo, para Deleuze, nunca es autónomo y aislado, nunca se distingue de su entorno. Por el contrario, el individuo está inmerso en un medio que está implícito en todo lo que hace, en todas las facetas de su modo de vida. Cuando Deleuze sostiene que su medio está implícito en su vida, es porque sus acciones están determinadas por los afectos a través de los cuales interioriza sus relaciones con el mundo.

6 "Deleuze plantea, a veces de manera absolutamente explícita, una vía distinta de interpretación de la tesis de la univocidad del ser, la de cierta filiación profunda o copertenencia entre lenguaje y ontología" (Hernández, 2004, p. 127). 
genuina. Deleuze explica en Diferencia y Repetición que el Ser "se dice en un mismo sentido de todas, pero ellas mismas no tienen el mismo sentido" (Deleuze, 2006: 72). Ahora bien, Spinoza trata este mismo concepto de ser unívoco de pura afirmación, en lugar de neutralidad o indiferencia, ya que es "igual y esta inmediatamente presente en todas las cosas (...) la univocidad del ser significa, pues, también, la igualdad del ser" (Deleuze, 2006: 74-75). De hecho, Spinoza tanto como Deleuze dicen que, no puede haber varias sustancias que compartan un atributo común, sino que puede haber una sola Sustancia para todos los atributos, en este sentido, los atributos heterogéneos son pensamiento y extensión.

La univocidad espinozista juega un papel muy importante en la formulación de Deleuze sobre el concepto de inmanencia. La filosofía de la inmanencia de Deleuze está influenciada de manera indispensable por la ontología de Spinoza, que no es meramente materialista, sino también panteísta, atea, pero de naturaleza ética. La ontología espinozista es panteísta, porque la naturaleza y Dios son comprendidos como idénticamente responsables de la producción de todos los afectos ${ }^{7}$, es decir, que pone énfasis en las experiencias corporales. Es atea ya que denuncia la conciencia, los valores y las pasiones tristes y, lo que es más importante, a un Creador fuera del ser.

Por último, es ética puesto que Spinoza, en la percepción de Deleuze, nos abre a una vida de pura inmanencia:

Se dirá de la pura inmanencia que es UNA VIDA, y ninguna otra cosa. No es inmanencia a la vida, pero lo inmanente que no es en nada es él mismo una vida. Una vida es la inmanencia de la inmanencia, la inmanencia absoluta: es potencia, beatitudes completas (Deleuze, 2002: 234).

En una vida de univocidad, el mundo se convierte en un plano democratizado para todos los seres. Fuera del universo espinozista, el tema filosófico de la inmanencia es paralelo a la noción del todo de Bergson como constitutivo del tiempo monista, "para Bergson la duración no era simplemente lo indivisible o lo no-mesurable, sino más bien lo que se dividía cambiando la naturaleza" (Deleuze 1987,38), las duraciones infinitas y la virtualidad pura del trágico mundo de Dionisos de Nietzsche. Para el primero, el Todo es un plano donde el espacio, la duración, la percepción. la memoria, el pasado y el presente; la memoria de recuerdo y la memoria de contracción se entienden en el criterio de la coexistencia virtual, es decir, "lo que esta percepción tan especial expresa ya no es entonces una acción virtual, sino una acción real: la afección consiste en esto mismo (Bergson 2010,74). Para este último, el mundo divinizado es un mundo de puro devenir cuyo principio rector es el Eterno Retorno ${ }^{8}$.

Deleuze explica que el título del último capítulo, "Spinoza y nosotros" de su libro, Spinoza: Filosofía práctica, qué significa estar en medio de Spinoza. Precedente a la

\footnotetext{
7 "Hay tantasclases de alegría, tristeza y deseo y, consiguientemente, hay tantas clases de cada afecto compuesto de ellos —como la fluctuación del ánimo-, o derivado de ellos —amor, odio, esperanza, miedo, etc.—, como clases de objetos que nos afectan" (EIII, Prop,56).

${ }^{8}$ Deleuze define el Eterno Retorno como "el ser de lo que deviene. «Decir que todo retorna es aproximar al máximo el mundo del devenir y el del ser" (cf. Deleuze, 2013, p. 71).
} 
caracterización de Deleuze de la micropolítica como una política del medio, es la recomendación de que la forma más profunda de comprender el espinozismo es a través del medio. En sus palabras:

Generalmente uno comienza con el primer principio de un filósofo. Pero lo que cuenta también es el principio tercero, cuarto o quinto. Todos conocen el primer principio de Spinoza: una sola sustancia para todos los atributos. Pero también conocemos el tercer, cuarto o quinto principio: una sola Naturaleza para todos los cuerpos, una sola Naturaleza para todos los individuos, una Naturaleza que es ella misma un individuo capaz de variar en un número infinito de maneras. (Deleuze, 2004: 149).

Una filosofía del medio exhibe la reconstrucción radical de Deleuze del monismo de Spinoza. No se trata simplemente de una afirmación conservadora de una sola sustancia, sino más bien de un diagrama de un plano común de inmanencia donde todos los cuerpos, mentes e individuos se sitúan de diferentes maneras (cf. León, 2019). En este ámbito, el plano de organización ${ }^{9}$ o trascendencia y el plano de inmanencia no se perciben como dos opuestos, sino como una multiplicidad de dimensiones, líneas y direcciones en un agenciamiento (cf. Deleuze, 2002: 238). La legitimidad del primero emana de una esfera trascendental: un plan divino en la mente de Dios y una evolución en la organización del poder de una sociedad. El plano de la organización siempre involucra formaciones de subjetividad estructural, que solo se pueden inferir de lo que es dado. El plano de inmanencia, por otro lado, se compone de innumerables colectividades, individuos y cuerpos, sujetos a combinaciones o relaciones multifacéticas. Deleuze piensa que el plano de inmanencia es el plano de composición de la naturaleza.

En este sentido, infundir ideas en medio de Spinoza implica dos cosas. Primero, involucra a Spinoza, incluida la creencia subyacente de Deleuze de que el pensamiento carece de cualquier origen primordial, pero solo del exterior por el cual está conectado; $y$, segundo, estar en medio de Spinoza significa ubicarse en el plano modal inmanente. $Y$ dado que este plano no se basa en ningún principio metafísico, las fuerzas de relación y los cuerpos se definen de acuerdo con sus desempeños y variaciones materiales, es decir, en su capacidad de afectar y ser afectados por otros cuerpos, ya sea en virtud de construcción o de descomposición. La incesante transformación del plano de inmanencia, fragmenta el trabajo normativo del plano de organización que, a su vez, redobla y re-expresa esta desolación al intentar bloquear y contener en formas molares la movilidad y el dinamismo de lo molecular.

La inmanencia es un término muy significativo y general en todo el canon deleuziano. La teorización de la inmanencia de Deleuze, muy influenciada por la naturaleza de Spinoza, es perceptible en su posición antijurídica contra la filosofía del

\footnotetext{
9 "Orden social o político aparato de estado. "De los muchos sentidos de la palabra "plano", nos concentraremos brevemente en una de las distinciones centrales dentro de la filosofía de Gilles Deleuze, distinción que recae entre tres "espacios" Ilamados "plano de inmanencia", "plano de consistencia" y "plano de organización". Los tres planos no se dan por separado, sino que muy bien pueden aparecer simultáneamente en cualquier operación de desterritorialización" (Bogado).
} 
Estado. La filosofía anti-jurídica de Deleuze es un apéndice de su crítica general a la moralidad trascendental, la moral responsable de la marginación de la materialidad de la vida y las potencialidades creativas del cuerpo. Spinoza es un testigo crítico de este tipo de moralidad prevalente en el saber occidental, especialmente en la tradición jurídica. En esencia, la erudición jurídica implica que las fuerzas tengan un origen individual o particular; que deben ser socializados para generar una relación que les corresponda adecuadamente.

Hobbes, mucho antes que Kant, "tiende a un idealismo si no trascendental al menos suficiente como para sacar a Hobbes del grupo de los empiristas y ponerlo más bien cerca de los racionalistas como Leibniz, Spinoza o Kant" (Vargas, 2002:58). Para Hobbes, el poder del Estado está determinado por su poder de legitimación. El Estado configura su legitimidad como un garante metafísico basado en la voluntad racional de los individuos (Leviatán). Mientras que, Spinoza afirma que el derecho natural de un individuo se refiere simplemente a su acto de autoconservación conatus ${ }^{10}$. Frente a Hobbes, argumenta que el conatus de un individuo y los derechos del hombre no son transferibles a otro, especialmente a una autoridad metafísica. Del mismo modo, en el territorio hobbesiano, lo único común a cada individuo es su deseo de autoconservación, especialmente cuando existe un objeto de interés común ${ }^{11}$.En este sentido, parece que el poder del Estado está simplemente formado por su capacidad real o deseo de autoconservación que anula la idea de llegar a una racionalidad colectiva, que no es más que una idea caprichosa.

No obstante, para alejar a Spinoza de la acusación de anarquismo, se debe aclarar que no niega categóricamente ningún esfuerzo del Estado para armonizar las relaciones individuales en forma de estándares éticos y políticas públicas. A lo que se opone es al proyecto Estatal de avanzar en sus intereses totalizadores y narcisistas utilizando la estratagema de lograr la cohesión comunitaria bajo la autoridad del Leviatán es decir, "todos son políticos porque todos necesitan una audiencia, un espacio organizado públicamente" (Virno,2001: 13). En otras palabras, solo son rehusadas todas las representaciones trascendentales que se superponen por iniciativa de la multiplicidad de una síntesis trascendente.

Spinoza describe el estado, formulando una especie de horizonte inmanente caracterizado por fuerzas activas de relaciones y posibilidades, es decir, contra las diversas mistificaciones jurídicas del Estado, como un producto de un proceso puramente natural, en consonancia con la labor del derecho natural y la vida personal. En este sentido, la configuración genealógica del estado no es más que un producto de procedimientos y luchas secularizadas, y no de procesos metafísicos o cargados de divinidad. Por ejemplo, la relación ética y diferencial entre cuerpos, en el Estado se definen no de acuerdo con un principio trascendental general. Más bien, "es verdad

\footnotetext{
10 "Spinoza se dio cuenta antes que Nietzsche de que una fuerza no era separable de un poder de ser afectado, y que este poder expresaba su poder" (Deleuze 2013,11).

${ }^{11}$ El concepto de naturaleza humana Hobbes lo define estrictamente desde los supuestos del mecanicismo. De este modo, afirma que la esencia actual del hombre es su conatus, consistente en el esfuerzo por mantenerse en la existencia, de tal forma que el "fin principal del hombre es su propia conservación" (Hobbes, 1980:223).
} 
que somos siempre determinados; nuestro conatus mismo es determinado por las afecciones que experimentamos" (Deleuze, 1999: 240).

En el estudio de la etología, el comportamiento de los cuerpos en el plano de inmanencia se rige por el poder de la autoconservación. Sin embargo, la perseverancia de los numerosos cuerpos implica un encuentro necesario con otros cuerpos. Se puede argumentar, por lo tanto, que la aptitud y el movimiento de los cuerpos pueden variar dependiendo de la calidad y cantidad de otros cuerpos con los que se asocian. Del mismo modo, este encuentro creativo plantea la idea de que incluso el poder de la autoconservación no es inmune a los afectos del exterior. Esta caracterización inspiró a Spinoza a reconfigurar la democracia. Bajo la rúbrica del materialismo, la producción y la política de la multitud. Esta fase madura en la filosofía de Spinoza, desde el Tratado Teológico Político en adelante, constituye un materialismo radical de cuerpos y superficies donde la praxis constituye el ser como una reconstrucción incesante por la praxis humana.

\section{La etología como filosofía inmanente}

La ontología materialista de Spinoza es una etología o una ética situada en el plano de inmanencia. En este plano, solo hay una sustancia inmanente: el individuo humano, un modo de los atributos de la naturaleza y un fragmento de un todo variable e interrelacionado. Pero es importante acentuar que, en este plano, el individuo no disfruta de una posición privilegiada en relación con otras entidades, de la misma manera que la subjetividad no se limita al ámbito de la racionalidad. La univocidad del ser promueve una democracia ontológica, donde todo se metamorfosea en cuerpos, cuyo valor se mide no de acuerdo con las capacidades racionales y discursivas, sino con las velocidades, así como la capacidad de afectar y ser afectado. La misma descripción se encuentra en el trabajo colaborativo de Deleuze y Guattari, Mil Mesetas Capitalismo y Esquizofrenia (2002), donde afirman que, en el estudio de la etología, los cuerpos no se definen de acuerdo con algunas categorías lógicobiológicas como el género y la especie:

El problema no es en modo alguno el de los órganos y las funciones, y un Plan transcendente que sólo podría dirigir su organización bajo relaciones analógicas y tipos de desarrollo divergentes. El problema no es el de la organización, sino el de la composición; no es el del desarrollo de la diferenciación, sino el del movimiento y el reposo, la velocidad y la lentitud (Deleuze y Guattari, 2002: 259).

De igual manera, en El Anti-Edipo Capitalismo Y Esquizofrenia (2016), consideran que la etología puede ser comprendida en el ámbito de la producción de deseos. En este espacio, no existe una dicotomía entre el hombre y la naturaleza, o lo humano y lo no humano. En lugar de percibirlos como opuestos binarios, se perciben más bien, como parte de un todo en el proceso de vida.

Pero no el hombre como rey de la creación, sino más bien como el que llega a la vida profunda de todas las formas o de todos los géneros, como hombre cargado de estrellas y de los propios animales, que no cesa de empalmar una máquina-órgano a una máquina-energía (Deleuze y 
De esta forma, Deleuze explica cómo el concepto de inmanencia en el universo espinozista logra un estado ontológico. La sustancia, los atributos y los modos se entienden en relación con la expresión, más que con una organización trascendente de emanación. La sustancia no tiene privilegios en relación con ningún atributo, de la misma manera que ningún atributo es superior a otro. Un atributo expresa una esencia particular. Como argumenta Deleuze "Todos los atributos formalmente distintos son relacionados por el entendimiento a una substancia ontológicamente. Pero el entendimiento sólo reproduce objetivamente la naturaleza de las formas que aprehende. Todas las esencias formales forman la esencia de una substancia absolutamente una" (Deleuze, 1999: 59).

Ahora bien, en Spinoza y el problema de la Expresión, Deleuze aclara el concepto de expresión como dispositivo hermenéutico de doble filo que nos habilita para interpretar textos, relaciones y acontecimientos, no limitados a las fronteras de representación, identidad, linealidad y teleología. Al comprender la historia, por ejemplo, no debemos interpretarla simplemente como una progresión lineal de eventos hacia un gran propósito o simplemente como la culminación de un concepto unitario único. Este nuevo tipo de filosofización histórica no se ajusta a ninguno de los dualismos mencionados anteriormente, sino que opera en el medio.

La historia como historicismo y el devenir nómada, el expresionismo desmantela la estratificación lógica y el esquema arbóreo de las cosas hacia una lectura relacional y minortariana o intermedia de comprender las cosas, en un nivel paralelo de expresión. En el nivel macro, ser espinozista implica abrazar la vida desnuda en sus aspectos ocultos, inconscientes y marginales en la historia de la filosofía: "Hay un devenirfilósofo que no tiene nada que ver con la historia de la filosofía, y que pasa más bien por todos aquéllos que la historia de la filosofía no logra clasifica" (Deleuze y Parnet, 1980: 6). De hecho, una noción deleuziana de inmanencia se desarrolla como una especie de ontología heterogenética creativa que extiende el concepto de expresionismo de Spinoza al representar cómo la sustancia produce sus propios modos y características a través de un doble proceso de diferenciación.

Al mismo tiempo, la etología es una perspectiva filosófica que no presupone una realidad más allá de los contornos de las materialidades de la vida y es irreductible a los regímenes epistemológicos de la metafísica platónica, cartesiana y kantiana. Dado que la metafísica es la doncella de la moralidad, la etología de Spinoza se transforma en una ética antitética y ontológica de singularidades y devenires. Además, debido a que el lugar de la moral es el sujeto universal o el organismo mecanicista, la etología sirve como un lugar para una micropolítica del sujeto que se enfoca en las fisuras moldeadas de su incesante relación consigo mismo y con otros agenciamientos de cuerpos. Hablando de agenciamiento de cuerpos, el cuerpo asume la posición de ser el nuevo modelo de filosofar en el estudio de la etología. En, La Ética, Spinoza afirma que: "el hecho es que nadie, hasta ahora, ha determinado lo que puede el cuerpo, es decir, a nadie ha enseñado la experiencia, hasta ahora, qué es lo que puede hacer el cuerpo en virtud de las solas leyes de su naturaleza" (EIII, Prop.II. Escolio II). Si el cuerpo es animal o humano realmente no es el punto. En lugar, la ética está ligada a los límites y capacidades de ese cuerpo. Según Deleuze y Guattari, debemos llegar a 
saber qué puede hacer un cuerpo, su potencial para interactuar con otros cuerpos, si esa interacción traerá o no daño a cualquiera de los cuerpos y si existe el potencial de intercambio o unión para formarse un cuerpo aún más fuerte

La adaptación de Spinoza para hacer del cuerpo como el nuevo modelo significa su capacidad para superar todas nuestras abstracciones lógicas relacionadas con él, incluida la capacidad de los pensamientos para ir más allá de la conciencia. Deleuze argumenta que:

Hay menos cosas en el espíritu que superan nuestra la conciencia, que cosas en el cuerpo que exceden nuestro conocimiento. Solo por un único e igual movimiento que llegaremos, si es que es posible, a captar el poder del cuerpo más allá de las condiciones dadas de nuestro conocimiento ya a captar la potencia del espíritu más allá de las condiciones dadas de nuestra conciencia (Deleuze, 2004: 28).

Los diversos poderes y posibilidades del cuerpo solo pueden desatarse a través del sincretismo perpetuo y las luchas con otros cuerpos. Esto inspira a Spinoza a desarrollar el concepto de agencia ${ }^{12}$. La razón detrás de la formulación de la agencia de Spinoza es doble puesto que, históricamente, busca revolucionar la multitud decadente de su tiempo; y filosóficamente, aspira a antagonizar la noción tradicional o trascendental de subjetividad que formó enormemente el pensamiento filosófico occidental. Su idea de agencia apunta críticamente a salvar al cuerpo de su marginación en la historia de la moral occidental. Desde la antigüedad, el cuerpo está subordinado a la lógica de la mente o la conciencia. Sus atributos contingentes, como la corporeidad y la mutabilidad, son considerados como debilidades de la condición humana sin explorar minuciosamente sus grandes e irrepetibles potencialidades. En general, esta violencia histórico-filosófica representa la supremacía de la filosofía trascendental sobre la inmanencia en toda la erudición filosófica occidental, aparentemente reflejada en la moralidad.

La agencia de Spinoza está integralmente encaminada por la dinámica de la materialidad de la vida. Este atributo radical influyó en la apropiación de Deleuze del concepto de agencia como algo colectivo y agenciado. Una apropiación deleuziana de la agencia espinozista tiene que ver con los procesos de colectivización que producen la composición o combinación de individuos con mayor poder y multiplicidad, e individuos como modalidades de estos individuos mayores.

un Colectivo (agenciando elementos, cosas, vegetales, animales, herramientas, hombres, potencias, fragmentos de todo eso; pues no puede hablarse de "mi" cuerpo sin órganos, sino de "yo" en él, lo que queda de mí, inalterable y cambiando de forma, franqueando umbrales) (Deleuze y Guattari, 2002: 166).

\footnotetext{
${ }^{12}$ En la filosofía de Spinoza, la autoconfianza es, necesariamente, un correlato emocional de la capacidad de agencia "a capacidad de agencia coincide tautológicamente con la libertad en sentido espinozista, y finalmente, el conocimiento de las causas de la propia situación no es otra cosa que el desarrollo cognitivo espinosista, la expansión del ser, que constituye el corazón de la propuesta ética espinosista" (Caraballo, 2012:212).
} 
El encuentro de agencias "organismos" puede aumentar o disminuir sus capacidades y puede crear constelaciones, afectos e intensidades novedosas o degeneradas. Nietzsche llama a este evento como la experiencia del caos dentro de uno mismo que no solo implica una relación reflexiva con uno mismo, sino también la ruptura egoísta o trascendental como una condición previa para un devenir-vida. En la agencia humana espinozista, el individuo está dispuesto a asociarse activa o pasivamente con otros cuerpos, lo que fortalecerá su capacidad de recuperación. Siguiendo a Deleuze. "nuestros sentimientos o afectos brotan del encuentro con otros modos existentes" (Deleuze, 2004: 64), En otras palabras, el modo de existencia de una agencia individual puede ser considerado como malo, servil o débil, si él o ella está incapacitado para ejercer su poder de actuación. Y debe considerarse como bueno, libre o racional cuando él o ella exhibe su capacidad de ser afectado en donde su poder de actuación aumenta y se fortalece, creando así afectos activos e ideas adecuadas "se considerará que un modo de existencia es bueno o libre, racional o fuerte cuando ejerce su capacidad de ser afectado. A su vez Deleuze emplea el concepto de variaciones para servir a su oportuna apropiación filosófica del afecto" (León, 2018: 196).

Sin embargo, la complejidad sigue siendo que las consecuencias creativas y nobles no encuentran garantía y transparencia en un mundo de inmanencia porque no hay atributos predeterminados de la mente, el cuerpo y las relaciones. Dado que la constelación de cuerpos puede fomentar igualmente afectos no deseados o degradados, Spinoza formula la teoría de nociones comunes o compactas para regular o condicionar encuentros y posibilidades que afirman la vida. De acuerdo con Deleuze "mientras nuestros sentimientos o afectos brotan del encuentro con otros modos existentes, se explican por la naturaleza del cuerpo afectante y de la idea y por la idea necesariamente inadecuada de este cuerpo" (Deleuze, 2004: 64). Sugiere que, con la ayuda de pasiones alegres, se crea la idea de lo que es común a nosotros y a los cuerpos externos a nosotros. En otras palabras, el acuerdo o la unidad entre dos o más cuerpos es coextensivo con la utilidad, es decir, cuando dos cuerpos chocan por la preocupación común de preservar su composición y la escasez de recursos, se transforman en enemigos. No obstante, en lugar de interpretar este evento de encuentro de cuerpos de una manera hobbesiana, siguiendo a de Deleuze se puede abordar esta problemática de manera diferente, puesto que, dos o más cuerpos deben reconocer que el pacto tiene un interés mutuo racional a largo plazo.

Ahora bien, siguiendo estos argumentos podemos decir que: en primer lugar, todos los hombres tienen una naturaleza relevante similar, estas naturalezas son agradables, por lo que cada uno se verá afectado de alegría en el encuentro. En segundo lugar, esto significa que la cantidad de pasiones activas se incrementará en cada individuo y el poder de acción del individuo aumentará. Esto es en interés de cada individuo. Finalmente, los cuerpos individuales se unen y es a través de esta tercera relación que componen un solo cuerpo. Aunque cada cuerpo se conserva en la nueva combinación, el nuevo cuerpo es dos veces más fuerte. Además, una idea tan adecuada de la noción común nos permite estar en posesión de nuestro poder de acción, porque:

El espíritu que forma una idea adecuada es causa adecuada de las ideas 
que derivan de ésta: es en ese sentido que es activo (...) Las pasiones dichosas son las ideas de las afecciones producidas por un cuerpo que conviene con el nuestro; nuestro espíritu sólo forma la idea de lo que es común a ese cuerpo y al nuestro (Deleuze, 1999: 277).

En el contexto más amplio de las relaciones políticas, la etología busca proporcionar una base desde la cual repensar a lo político de una manera no hegemónica o no normativa que evadiría la reducción de variaciones o diferencias en las relaciones de opresión y dominación. El individuo humano, en el estudio de la etología, se configura a lo largo de dos ejes: cinético y dinámico. En el primero, el individuo se percibe como compuesto de otros cuerpos y cuyo atributo es relacional (velocidad y lentitud) en la naturaleza. En este último, el individuo es visto como una cadena de poder en el mar de otros cuerpos que pueden afectar y ser afectados por ese individuo.

Del mismo modo, la identidad del cuerpo se concibe como una realidad abierta debido a su incesante diálogo con el mundo exterior. Dado que el cuerpo es una realidad inmanente, sus configuraciones internas dependen de su capacidad para afectar y ser afectado por otros. De acuerdo con Deleuze podemos decir que "La estructura de un cuerpo es la composición de su relación. Lo que puede un cuerpo es la naturaleza y los límites de su poder de ser afectado (Deleuze, 1999: 209).

El individuo en la filosofía espinozista se entiende como la organización compleja del modo finito existente en cualquier atributo. Este modo tiene una esencia singular, que es también un grado de poder. Sin embargo, se caracteriza por la singularidad, la esencia del individuo es de naturaleza relacional. Deleuze aclara las potencialidades del cuerpo en términos de lo que Spinoza llama modos. Como un tipo de modalidad, el cuerpo se caracteriza por una esencia perceptible como una gradación de poder que contiene varias partes inmanentes. La expresión de una sola sustancia a través de modos solo lleva estas partes en virtud de su capacidad para afectar y verse afectado en una relación particular.

Debido a que los modos son derivados de relaciones interminables, simplemente disminuirá cuando ya no contenga esta habilidad, ya que ellos mismos son afectos activos, es decir, "un modo deja de existir cuando ya no puede seguir manteniendo entre sus partes la relación que lo caracteriza; igualmente, deja de existir cuando "ya no está apto para poder ser afectado de un gran número de manera" (Deleuze, 1999: 208). En resumen, el individuo está compuesto de partes extensas ilimitadas. Pero estas partes no son constitutivas de esencias individuales en sí mismas porque están organizadas solo sobre la base de un determinismo extenso. A pesar de las posibilidades gemelas de un cuerpo de descomponerse o fortalecerse después de un cierto encuentro con otro, el hecho es que, en cada relación, existe una verdad eterna.

En este punto, se debe recalcar que, a pesar de la radicalización de la metafísica de Spinoza, él no defiende el privilegio del cuerpo sobre la mente o la conciencia, pero no quiere caer en un círculo vicioso de privilegio del cuerpo sobre la mente solo para dar justicia servil al primero. Deleuze escapa a estas arenas movedizas filosóficas al introducir el concepto de paralelismo:

Porque el alma y el cuerpo son estrictamente lo mismo bajo atributos 
diferentes. El alma y el cuerpo son la misma modificación de la sustancia en dos modos de atributos diferentes. Se distinguen por el atributo, pero son la misma modificación. Yo llamaría "alma" a una modificación relacionada con el atributo del pensamiento y "cuerpo" a la misma modificación bajo el atributo de la extensión. De allí la idea de un paralelismo del alma y del cuerpo: lo que el cuerpo expresa en el atributo de la expresión, el alma lo expresa en el atributo del pensamiento (Deleuze, 2008: 150).

Su idea de paralelismo demuestra que el cuerpo excede los registros epistémicos que tenemos de él, de la misma manera que el pensamiento excede la conciencia que tenemos de él. Esta es la razón por la cual Deleuze sugiere que la noción tradicional de la filosofía de la conciencia debe repensar su arrogancia y ceguera en relación con el cuerpo. La conciencia es causada por afectos determinados: la conciencia del conato sobre el esfuerzo de las cosas y el hombre por preservar su ser se produce por el afecto hacia la alegría o la tristeza, y desde los afectos "de estas composiciones y descomposiciones; experimentamos alegría cuando un cuerpo se encuentra con el nuestro y se compone con él" (Deleuze 2004: 29). Así, el principio de paralelismo se adhiere a la univocidad del ser y apunta al descubrimiento de un inconsciente del pensamiento tan profundo como lo desconocido del cuerpo.

Deseo y revolución por-venir

Ahora con estas reflexiones, es necesario interpretar la relación entre la ética inmanente de Spinoza y la teoría del deseo de Deleuze. En la agencia individual, el deseo como conatus ilustra al individuo según lo determinado por su poder de ser tanto el sujeto como el objeto de afecto siempre que no separemos la esencia de la acción, un conatus puede ser comprendido como la esencia de un ser en su grado de poder "Sin duda, una vez que existimos, nuestra esencia es un conatus, un esfuerzo de perseverar en la existencia" (Deleuze, 1999: 241). En los escritos maduros de Deleuze, el cuerpo (como conatus) se convierte en constitutivo de varias máquinas deseantes. Las máquinas deseantes son partes que no están relacionadas con ningún conjunto conectado a otras máquinas deseantes, algunas dentro del cuerpo, otras en el mundo natural y social. Al contrario de la devaluación del deseo del psicoanálisis convencional, Deleuze y Guattari describen al deseo como una fuerza revolucionaria y un proceso social de experimentación capaz de crear conexiones y sujetos nómadas que desafían la edipalización.

A nivel macro, Deleuze y Guattari enfatizan en el Anti-Edipo que el deseo o la producción deseante no pueden separarse de las producciones sociopolíticas. Esto da mérito a transfigurar la relación entre la ética de Spinoza y la teoría del deseo de Deleuze en una preocupación política, "el esquizoanálisis no oculta que es un psicoanálisis político y social, un análisis militante: y ello no porque generalice Edipo en la cultura, en las condiciones ridículas mantenidas hasta ahora" (Deleuze y Guattari, 2016: 104). Si la filosofía de la trascendencia simboliza la impotencia, la degeneración y la esclavitud, entonces una ética deleuziana investigaría y analizaría las condiciones que engendran a las personas que desean trascendencia u opresión. El libro Anti Edipo Capitalismo y Esquizofrenia, como producto filosófico de la protesta 
estudiantil de 1968, busca responder a este problema político. En el Prefacio ${ }^{13}$ de Foucault al libro mencionado, él señala a tres adversarios del AntiEdipo "el fascismo histórico de Hitler y Mussolini (...) sino también el fascismo que se halla dentro de todos nosotros, que acosa nuestras mentes y nuestras conductas cotidianas, el fascismo que nos hace amar el poder, desear aquello mismo que nos domina y explota" (Foucault, 1994: 2-3). Entre los tres, afirma que el enemigo más peligroso es el fascismo.

El deseo de trascendencia que se encuentra en las primeras publicaciones de Deleuze se transfigura en el deseo del fascismo o dominación en sus escritos maduros. Basado en el contexto sociohistórico de la revuelta estudiantil, El Anti-Edipo se actualiza como su crítica del instinto o deseo de la manada del pueblo francés, especialmente en la era posterior a 1968. Esto les permite conceptualizar su teoría ética o política del deseo inmanente como un antagonismo contra todos los análisis psicoanalíticos y sociopolíticos reductivos que permanecen configurados bajo el principio de trascendencia o edipalidad, hacia la multiplicidad del deseo.

La política de deseo de Deleuze y Guattari es colectiva y de agenciamiento, una micropolítica indispensablemente en deuda con la etología de Spinoza. A pesar de la estrecha afinidad de Spinoza y Deleuze con la filosofía antinihilista de Nietzsche, El Anti Edipo no personifica la existencia solitaria. La militancia, en el marco de Deleuze y Guattari, aprendería de la locura, pero luego iría más allá, de las desconexiones y desterritorializaciones, a nuevas conexiones. Una política del deseo vería la soledad y la depresión como las primeras cosas a seguir. La teorización positiva del deseo de Deleuze y Guattari es un factor integral en la conceptualización del principio del esquizoanálisis. Su objetivo principal es penetrar y desmantelar radicalmente los diversos conceptos, zonas y relaciones segmentadas que ya están editadas por el fascismo o en la actualidad. capitalismo avanzado.

La tarea del esquizoanálisis, va a través de la destrucción de todo un lavado del inconsciente, un legrado completo. Destruye Edipo, la ilusión del ego, la marioneta del superyó, la culpa, la ley, la castración "toda una limpieza, todo un raspado del inconsciente" (Deleuze y Guattari, 2016: 321). Lo que es más importante, aspira a buscar las constelaciones desterritorializadas del deseo ilesas o los flujos que no se han reducido a los códigos edípicos y las territorialidades neuroticizadas, las máquinas deseantes que escapan a tales códigos como líneas de escape que conducen a otros lugares. En otras palabras, el esquizoanálisis tiene como objetivo emancipar el deseo de ser totalizado por expresiones múltiples y multifacéticas de fascismo o Edipo. Deleuze y Guattari creen que el deseo del esquizoanálisis puede traducirse en una política radical que puede instigar a los individuos y colectividades contemporáneas a formular múltiples constelaciones, así como a convertirse en conductos dinámicos de fuerzas.

La política del deseo es una micropolítica, es decir, la micropolítica deleuziana se compone de tres líneas de intersección, a saber: la línea segmentada, la línea flexible

\footnotetext{
${ }^{13}$ Algunas ediciones en español del Antiedipo, no tienen este texto, del prefacio escrito por Foucault para este libro y esta como un texto separado, El Anti Edipo Introducción A La Vida No-Fascista.
} 
y la línea abstracta. La primera línea, la segmentación rígida, ilustra nuestra condición binaria, lineal y circular en la sociedad (cf. Deleuze y Guattari, 2016: 78). Las segundo, la línea molecular, representa una segmentaridad flexible, ya que están contextualizados, localizados y son similares a la web. Deleuze y Parnet afirman que las líneas moleculares son las grietas entre las segmentaciones o los flujos minoritarios con umbrales capaces de formar devenires rizomáticos y micro-devenires (cf. Deleuze y Parnet, 1980: 141). Por último, la tercera línea se llama línea abstracta. Es una línea capaz de escapar de segmentaciones y umbrales hacia destinos imprevisibles e intensidades nómadas. La última línea es la línea del desapego abstracto y el devenir absoluto o la desterritorialización. En resumen, las tres líneas se cruzan entre sí en todas las organizaciones, grupos e individuos.

Específicamente, la micropolítica es una búsqueda de la línea nómada que generaría una transformación genuina y revolucionaria (cf. Etchegaray, 2015). Cuando la segunda línea se metamorfosea como el principio general de la política, la inestabilidad es de alta posibilidad. Por supuesto, tampoco puede ser la primera línea porque la segmentaridad o la política segmentada es la fortaleza de la filosofía del Estado, la némesis de lo nómada. ¿Esto implica entonces que la tercera línea, la línea abstracta, es la línea nómada? En Mil mesetas, Deleuze y Guattari opinan que "desde el punto de vista de la micropolítica, una sociedad se define por sus líneas de fuga, que son moleculares" (Deleuze y Guattari, 2002: 220). Para evitar más confusión, debemos referirnos a nuestra caracterización primaria de la línea nómada como el símbolo creativo y radical de la transformación. De esta manera, parece que sería más adecuado delegar la nómada en el espacio perpetuamente cambiante y amorfo entre las líneas estriadas y flexibles. La micropolítica no rechaza ni la primera ni la segunda línea; se queda entre ellas. Por lo tanto, la micropolítica opera entre la actualización de la incesante transformación, la multiplicidad y la fluidez, así como la creación de organizaciones y colectividades, hacia un mapeo de un pueblo y el mundo por-venir.

Conclusión

La política de deseo de Deleuze y Guattari está vitalmente inspirada en la etología de Spinoza. La etología no es solo un principio evaluativo, sino también un principio selectivo, es decir, una cartografía. En este sentido, las agencias individuales y las colectividades se diagraman en el plano de la inmanencia, de tal forma que:

un cuerpo sólo se de- fine por una longitud y una latitud: es decir, el conjunto de los elementos materiales que le pertenecen bajo tales relaciones de movimiento y de reposo, de velocidad y de lentitud (longitud); el conjunto de los afectos intensivos de los que es capaz, bajo tal poder o grado de potencia (latitud) (Deleuze y Guattari, 2002: 264).

Una cartografía deleuziana establece la composición de un cuerpo individual y colectivo a través de sus habilidades extensivas e intensivas, así como su movimiento y poder de los afectos. Dado que los cuerpos son un compuesto de muchos otros cuerpos, que a su vez consisten en otros cuerpos, pueden constituir las partes extensas de conjuntos sociopolíticos más amplios, que también tienen sus respectivos atributos intensivos característicos. En resumen, la cartografía Deleuziana selecciona cuerpos 
individuales, colectivos, políticos, estéticos, económicos, revolucionarios, etc., y los diagnostica en términos de composición y descomposición.

La política del deseo de Deleuze implica una revolución cartográfica. En virtud del esquizoanálisis, debe diagramar de manera similar los cuerpos y las relaciones nómadas cuyos deseos escapan de la edipalización. Dado que el plano de inmanencia se caracteriza por el agonismo expresionista, las conexiones corporales y de agenciamiento son siempre fluidas. $\mathrm{Y}$ debido a que está abierto a todo tipo de constelaciones, la vigilancia siempre debe estar activa, especialmente cuando las conexiones están desactivadas para fomentar líneas creativas de fuga, así como adulteradas o polarizadas por formas más sutiles de fascismo. A la luz de la praxis revolucionaria contemporánea, la cartografía se transfigura en un mapeo y selección de conjuntos al estilo de la filosofía de la Nietzsche del eterno retorno como una doctrina ética o cuerpos a través de distinciones culturales, geográficas y epistemológicas, que están capacitados para desmantelar las relaciones existentes que fosiliza los movimientos, frustra las constelaciones y reifica la acción radical. Pero no debemos ser ajenos a la realidad de que en una época de la sociedad post-control donde el capitalismo ya es axiomático y exponencialmente de alta velocidad, esta tarea es como enfrentar lo imposible.

Por último, una revolución es un mapeo creativo y rizomático constitutivo de una colectividad diferencial y cultural que carece de cualquier esencia fija u objetivo unitario. Un estilo deleuziano de cartografía revolucionaria no es simplemente una marca reaccionaria de resistencia como la Revolución Comunista. Implica una ruptura epistemológica de nuestra vieja imagen del pensamiento, como Deleuze explica en Nietzsche y la Filosofía, así como la caotización de nuestro deseo y las relaciones totalizadas por Edipo. Si el término, propósito o utopía ocupa algún lugar en la discusión, es en forma de un principio diferencial y autorreflexivo que nos guiaría y nos empoderaría para crear incesantemente conceptos capaces de transformar el presente. Sin embargo, no hay garantía de que estos conceptos generen más libertad en el futuro, y que los revolucionarios de hoy no se metamorfoseen como conservadores u opresores del mañana, no debemos desanimar a los revolucionarios a trabajar incansablemente por una revolución por-venir.

\section{Bibliografía}

Bergson, Henry (2010): Materia y Memoria. Buenos Aires: Editorial Cactus.

Bogado, Fernando, "Deleuze y el problema de la expresión La ficción entre la proto-forma y el plano de la organización". En revista: Luthor. http://www.revistaluthor.com.ar/spip.php?article109\#nb10 consultado 12-012020.

Caraballo, Germán Ulises Bula (2012). "Spinoza: emponderamiento y ética de la composición". En: Universitas Philosophica_ 29 (58):197-215. 
Deleuze, Gilles (1987): El bergsonismo. Madrid: Catedra.

- (1999): Spinoza y el problema de la Expresión. Barcelona: Atajos

-(2002): "Últimos textos: El "yo me acuerdo" y La inmanencia de la vida (Introducción, traducción y notas de Marco Parmeggiani)". En: Revista internacional de filosofía, ISSN 1136-4076, № 7, 2002, págs. 219-237.

— (2004): Spinoza: filosofía práctica. Barcelona: Tusquets

-(2006): Diferencia y repetición. Traducción de María Silvia Delpy y Hugo Beccaecece. Buenos Aires: Amorrortu editores.

- (2008): En medio de Spinoza. Buenos Aires: Cactus.

—(2013): Nietzsche y la filosofía. Barcelona: Anagrama

Deleuze \& Guattari (2002): Mil mesetas Capitalismo y Esquizofrenia. Barcelona: Pretextos.

-(2016): El Anti Edipo Capitalismo y Esquizofrenia. Barcelona: Paidos.

Deleuze \& Parnet (1980): Diálogos. Valencia: Pre.Textos.

Del Río, Elena (2012): Deleuze and the Cinemas of Performance: Powers of Affection.

Edinburgh: Edinburgh University Press; Reprint edition.

Etchegaray, Ricardo (2015): "Deleuze como lector de Spinoza" En: Pensar con Deleuze.

Editorial Abierta FAIA, Academia Latinoamericana.

Foucault, Michel (1994): El Anti Edipo Introduccion A La Vida No-Fascista. En Zona Erogena \# $18 . \quad$ http://www.congresoed.org/wpcontent/uploads/2014/10/michel-foucault-prologo-a-antiedipo-1.pdf

Consultado 16-01- 2020.

Hobbbes, Thomas (1980): Leviatan. Madrid: Editora Nacional.

Hernández, Juan Pablo (2004): "La Univocidad Del Ser: Lenguaje Y Ontología En Gilles Deleuze" En: Universitas Philosophica 43, (pp. 123-144) diciembre 2004, Bogotá, Colombia.

Massumi, Brian (1995): "The Autonomy of the affect" in: Cultural Critique, No. 31, The Politics of Systems and Environments, Part II., pp. 83-109. 
Núñez, Amanda (2010): "Gilles Deleuze. Pensar el porvenir". Daimon. Revista Internacional de Filosofía, Suplemento 3, 107-115

León, E. A. L. (2011): Gilles Deleuze y el psicoanálisis. A Parte Rei: revista de filosofía, (75), 7. http://serbal.pntic.mec.es/AParteRei/leon75.pdf

León, E. (2018): "Gilles Deleuze hacia una ética inmanente del deseo". Revista Filosofía UIS, 17(2),doi:http://dx.doi.org/10.18273/revfil.v17n2-2018011

León León, Eduardo Alberto. (2019): Gilles Deleuze y el afecto a propósito del cine. Tesis de maestría, Flacso Ecuador.

Spinoza. Baruch (2017): Ética. Madrid: Alianza Editorial.

Spinozianas: Filosofía de los Márgenes: http://aluceromontano.blogspot.com/2012/06/spinozianas-natura-naturans-ynatura.html Consultado 12-01-2020.

Spinoza, Korte verhandeling van God, de mensch, en deszelvs welstand

https://www.kb.nl/themas/filosofiethemas/filosofie/benedictus-despinoza/spinoza-korte-verhandeling-van-god-de-mensch-en-deszelvswelstand Consultado 23-10- 2020.

Vargas, Jorge (2002): La metafísica oculta de Hobbes. Una contribucion al estudio del pensamiento moderno. Límite. Revista Interdisciplinaria de Filosofía y Psicología, (9),48-71. [fecha de Consulta 23 de Octubre de 2020]. ISSN: 07181361. Disponible en: https://www.redalyc.org/articulo.oa?id=836/83600903.

Virno, Paolo. (2001): A Grammar of the Multitude: For an Analysis of Contemporary Forms of Life. Semiotext(e) foreign agents series. 